\title{
SULL'AUMENTO DI TEMPERATURA NEL MANTELLO DELLA TERRA PER COMPRESSIONE ADIABATICA
}

\author{
Paolo Emilio Valle
}

1. - L'aumento della temperatura nell'interno della Terra, dovuto a compressione adiabatica, ha una notevole importanza in diverse questioni di carattere geofisico $\left(^{(1)}\right.$.

La sua stima è stata eseguita da diversi autori, estrapolando all'interno della Terra $i$ valori del rapporto fra la dilatazione termica e il calore specifico a pressione costante, determinati in laboratorio per varie rocce ignee.

Solo recentemente è stato fatto un tentativo per una valutazione in base alla teoria dei solidi $\left({ }^{2}\right)$.

Questa nota ha lo scopo di mostrare come si possa pervenire facilmente ad una relazione che lega la densità, la temperatura e la velocità delle onde longitudinali e trasversali di un solido ideale, sottoposto ad una particolare trasformazione adiabatica e a quali risultati conduce la sua applicazione all'interno della Terra.

2. - E noto che l'agitazione termica in un solido si può pensare dovuta ad onde longitudinali e trasversali completamente diffuse $\left(^{(3-5}\right)$, per le quali esiste un limite inferiore alla lunghezza d'onda, in conseguenza della struttura discontinua del solido stesso. Lo spettro delle frequenze dei due tipi di onde è quindi limitato superiormente.

Se si usano gli indici $l$ e $t$ per distinguere le grandezze che si riferiscono rispettivamente alle onde longitudinali e trasversali, la frequenza limite per ciascun tipo di onda è data da

$$
v_{m}=v_{m}\left(\frac{3 V}{4 \pi V}\right)^{1 / 3}{ }_{(m=l, t)}
$$

dove $v_{\mathrm{m}}$ è la velocità, supposta indipendentemente dalla frequenza, ed $N$ il numero di atomi contenuti nel volume $V$.

Un solido così definito, cioè come un sistema continuo che ammetta un limite superiore per le frequenze delle onde elastiche, è il solido isotropo ideale. 
Il numero complessivo delle oscillazioni è uguale ad $N$ per le onde longitudinali e a $2 N$ per le onde trasversali. Pertanto, il solido va trattato come un sistema di $3 N$ oscillatori armonici, dei quali $N$ hanno la frequenza limite $v_{1}$ e $2 N$ la frequenza limite $v_{\mathrm{v}}$. In questa schematizzazione la meccanica quantistica permette il computo statistico dell'entropia $S\left({ }^{3-5}\right)$. La sua variazione elementare risulta data da

$$
d S=R \Sigma_{\mathrm{m}}\left(\frac{d D}{l X_{\mathrm{m}}}-\frac{L^{\prime}}{X_{\mathrm{m}}}\right) d X_{\mathrm{m}}{ }_{(\mathrm{m}=l, \mathrm{t}, \mathrm{t})}
$$

nella quale $R$ è la costante dei gas e

$$
D\left(X_{\mathrm{m}}\right)=\frac{3}{X_{\mathrm{m}}{ }^{2}} \int_{0}^{X_{\mathrm{m}}} \frac{\xi^{3} d \xi}{e^{\xi}-1}+\frac{3}{8} X_{\mathrm{m}}
$$

Se si introducono le temperature di Dehye $\Theta_{m}$, ossia si pone

$$
\Theta_{\mathrm{m}}=\frac{h v_{\mathrm{n}}}{k}
$$

$$
(m=l, t)
$$

dove $h$ è costante di Plank e $k$ la costante di Boltzmann, risulta

$$
X_{\mathrm{m}}=\frac{\AA_{\mathrm{m}}}{\bar{T}}
$$

essendo $T$ la temperatura assoluta.

Il secondo membro della [2] è quindi funzione della temperatura assoluta e del volume tramite le $\Theta_{m}$.

Si supponga ora di eseguire sul solido una trasformazione adiabatica reversibile, sottoponendolo ad una variazione isotropa ed omogenea di volume.

Si avrà

$$
\Sigma_{\mathrm{m}}\left(\frac{d D}{d X_{\mathrm{m}}}-\frac{D}{X_{111}}\right) d X_{\mathrm{m}}=0
$$

Se la temperatura $T$ è molto inferiore alle temperature di Debye, cioè $X_{m}$ è molto grande, si ha

$$
D\left(X_{\mathrm{m}}\right) \simeq \frac{3}{8} X_{\mathrm{m}}+\frac{\pi^{4}}{15} \frac{1}{X_{\mathrm{m}}^{3}}
$$

e si ottiene, tramite le [6], [5], [4] ed [1], 


$$
\frac{T^{3}}{\rho}\left(\frac{1}{v_{1}^{3}}+\frac{2}{v_{t}^{3}}\right)=\text { costante }
$$

nella quale $\varrho$ è la densità, mentre se $X_{m}$ è piuttosto piccolo si può usare lo sviluppo

$$
D\left(X_{\mathrm{m}}\right)=1+\frac{X_{\mathrm{m}}{ }^{2}}{20}-\frac{X_{\mathrm{m}}{ }^{4}}{1680}+\cdots
$$

e risulta in modo analogo, per $\Theta_{\mathrm{m}}<<\sqrt{20} \mathrm{~T}$,

$$
\frac{T^{3}}{\varrho v_{1} v_{1}^{2}}=\text { costante }
$$

E molto importante il fatto che la [8] e la [10] siano indipendenti dalla composizione chimica del solido. La [8] e la [10] si identificano se si pone $v_{1}=\sqrt{3} v_{1}$.

3. - Nell'interno della Terra, il valore della temperatura di Dehye relativa alle onde longitudinali $\dot{e}$ presumibilmente intorno ai $1700^{\circ} \mathrm{K}$ alla profondità di $1000 \mathrm{~km}$ e ai $2000^{\circ} \mathrm{K}$ alla profondità di $2898 \mathrm{~km}$, ossia alla base del mantello. Le temperature di Debye, relative alle onde trasversali, sono circa $1 / \sqrt{3}$ volte le precedenti.

É noto che fra queste due profondità si può ritenere che $i$ materiali presentino l'omogeneità richiesta per l'applicazione della [8] o della [10], e che l'andamento della densità sia adiabatico ( ${ }^{6-7}$ ).

I presunti valori delle temperature di Debye sopracitati in relazione al probabile valore della temperatura esistente alla profondità di $1000 \mathrm{~km}$, richiedono l'uso della [10].

In base alle grandezze contenute nella tabella $I\left({ }^{6-7}\right)$,

\section{Tabella I}

\begin{tabular}{|c|c|c|c|}
\hline $\begin{array}{c}\text { Profondità } \\
\mathrm{km}\end{array}$ & $\begin{array}{c}v_{1} \\
\mathrm{~km} \mathrm{sec}-1\end{array}$ & $\begin{array}{c}\mathrm{vm}_{\mathrm{t}} \\
\mathrm{km} \mathrm{sec}\end{array}$ & $\begin{array}{c}Q \\
\mathrm{gcm}^{-3}\end{array}$ \\
\hline 1000 & 11,42 & 6,36 & 4,68 \\
2600 & 13,50 & 7,21 & 5,54 \\
2898 & 13,64 & 7,30 & 5,68
\end{tabular}


dalla [10] si ottiene

Dalla [8] si otterrebbe

$$
T_{2898}=1,24 T_{1000}
$$

$$
T_{2898}=1.23 T_{1000}
$$

Cioè la temperatura alla base del mantello sarebbe circa 1,24 volte la temperatura a $1000 \mathrm{~km}$, se il materiale fosse stato compresso adiabaticamente.

E interessnte il confronto con il calcolo eseguito da J. Verhoogen $\left({ }^{2}\right)$.

La [8] e la [10] forniscono rispettivamente

$$
\begin{aligned}
& T_{2000}=1,20 T_{1000} \\
& T_{2000}=1,22 T_{1000}
\end{aligned}
$$

mentre il predetto autore ottiene

$$
T_{280}=1,18 T_{1000}
$$

Roma - Istituto Nazionale di Geofisica - Dicembre 1951

\section{RIASSUNTO}

Viene stabilita una relazione fra la densità, la temperatura e la velocità delle onde longitudinali e trasversali relativa ad una particolare trasformazione adiabatica di un solido ideale.

L'equazione, applicata al mantello della Terra, conduce al risultato che la temperatura in prossimità del nucleo sarebbe circa 1,24 volte la temperatura alla profondità di $1000 \mathrm{~km}$, se la compressione dei materiali fosse avvenuta adiabaticamente.

\section{BIBLIOGRAFIA}

(1) Benfield A. E.: Temperature in an accreting Earth. Trans. Amer. Geoplyys Union 31, 53.57 (1950).

(-) Verhoogen J.: The Adiabatic Gradient in the Mantle. Trans. Amer. Geophys. Union 32, 41.43 (1951).

(3) Brillouin L.: Teuseurs en Mécanique et en Élasticité. Masson, Paris (1946).

(4) Britlouin L.: Wave Propagation in Periodic Structures. Me Gran-Hill. New York (1946).

(5) Slater J. C.: Introduction to Chemical Physics. Mc. Gran-Hill. New York (1939).

(6) Bullen K. E.: The Problem of the Earth's Density Variation. Bull. Seism. Soc. Am. 30, 235-250 (1940).

(7) Bullen K. E.: Introduction of the Teory of Seismology. Cambridge University Press (1947). 